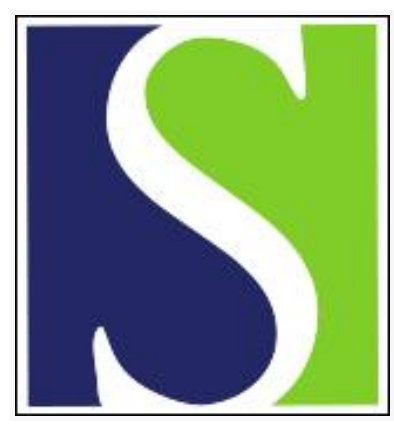

Scand J Work Environ Health 1992;18(6):337-345

https://doi.org/10.5271/sjweh.1564

Issue date: 01 Dec 1992

Impact of occupations and job tasks on the prevalence of carpal tunnel syndrome.

by Hagberg M, Morgenstern $\mathrm{H}$, Kelsh M

Affiliation: National Institute of Occupational Health, Division of Work and Environmental Physiology, Solna, Sweden.

The following articles refer to this text: 2020;46(1):43-49;

2020;46(6):618-629

This article in PubMed: www.ncbi.nlm.nih.gov/pubmed/1485158 


\title{
Impact of occupations and job tasks on the prevalence of carpal tunnel syndrome
}

\author{
by Mats Hagberg, MD', Hal Morgenstern, $\mathrm{PhD}^{2}$, Michael Kelsh, $\mathrm{MPH}^{2}$
}

\begin{abstract}
HAGBERG M, MORGENSTERN H, KELSH M. Impact of occupations and job tasks on the prevalence of carpal tunnel syndrome. Scand $J$ Work Environ Health 1992;18:337-45. In this investigation reported epidemiologic studies on carpal tunnel syndrome (CTS) (15 cross-sectional studies involving 32 occupational or exposure groups and six case-referent studies) were reviewed. The prevalence of CTS in the different occupational groups varied between 0.6 and $61 \%$. The highest prevalence was noted for grinders, butchers, grocery store workers, frozen food factory workers, platers, and workers with high-force, high-repetitive manual movements. Odds ratios greater than 10 were reported for exposed groups in three studies. On the basis of epidemiologic and other evidence, it was concluded that exposure to physical work load factors, such as repetitive and forceful gripping, is probably a major risk factor for CTS in several types of worker populations. At least $50 \%$, and as much as $90 \%$, of all of the CTS cases in these exposed populations appeared to be attributable to physical work load.
\end{abstract}

Key terms: entrapment, epidemiology, ergonomic, hand, nerve, neuropathy, occupational health, work load, wrist.

The association between occupational activities and carpal tunnel syndrome (CTS) has been addressed by several investigators. Despite the reported association between occupation or occupational exposure and CTS, a causal relationship between usage and CTS has been refuted. Recently an editorial in the Journal of Occupational Medicine claimed that "CTS is not a cumulative trauma disorder [p 39]." In the Newsletter, Occupational Problems In Medical Practice the same author claimed that "I am convinced that some forms of usage can exacerbate the symptoms of some regional musculoskeletal illness, but not all - for example, not CTS [p 7]" (2). On the other hand Gerr et al (3), in a review of upper-extremity musculoskeletal disorders of occupational origin, concluded that "carpal tunnel syndrome is etiologic related to occupational exposures [p 562]." Evidence for a causal relationship between workplace ergonomic factors and nerve entrapment of the median nerve at the wrist was recently reported in a meta-analysis by Stock (4). To infer a causal relationship between occupational exposure and CTS, epidemiologic studies would have to show a statistical association between occupation and CTS that is not due entirely to estimation errors (chance or bias).

The aim of the present investigation was to review

\footnotetext{
1 National Institute of Occupational Health, Division of Work and Environmental Physiology, Solna, Sweden, and Department of Occupational Health, Karolinska Hospital, Stockholm, Sweden.

2 Department of Epidemiology and Center for Occupational and Environmental Health, University of California at Los Angeles (UCLA), School of Public Health, Los Angeles, California, United States.
}

Reprint requests to: Professor M Hagberg, National Institute of Occupational Health, IFM, S-171 84 Solna, Sweden. the epidemiologic literature for the possible effect of occupation, specifically physical work load, on the occurrence of CTS and to consider the overall evidence for a causal relationship.

\section{Materials and methods}

A survey of the Medlars documentation system was performed for the years 1966 to 1990 (November); 164 references were obtained with the use of the key words carpal tunnel syndrome, CTS, occupation, incidence, and prevalence. Most of the articles dealt with case descriptions, diagnosis, or treatment $(60 \%$ of the references). Reviews or educational articles came second ( $20 \%$ of the references). Only $15 \%$ of the articles were population-based studies of CTS in occupational groups. Furthermore, we studied the reference lists of these articles to assess all the literature of interest for epidemiologic aspects of CTS. Only articles or official reports in which CTS was defined by both symptoms and signs were considered. These signs included an electrodiagnostic test of median nerve block, a positive Phalen's test, or a positive Tinel test. Surgical release of the median nerve at the wrist was also accepted as CTS, since both symptoms and signs are a general requirement for carpal tunnel surgery (5). Furthermore we included a study of occupational groups in which only nerve conduction was measured (6). Prevalence odds ratios with $95 \%$ confidence intervals were estimated for the different exposures or occupational groups in the studies in which the authors did not perform these computations (7). The prevalence odds ratio approximates the incidence rate ratio if the mean duration of the disease is the same for the exposed and the unexposed and no other sources of bias are present $(8,9)$. 
The impact of the exposure (occupational group or job title) was estimated by the attributable fraction in the exposed population - ie, the proportion of exposed cases that would not have developed the disease in the absence of exposure. In the reviewed studies the reference group had a different occupation or job task than the study group. The reference groups could not be regarded as unexposed but rather as less exposed than the study groups. Therefore the preferred terminology is reference group and not control group since exposure was not under control. The attributable fraction was estimated by $(\mathrm{OR}-1) / \mathrm{OR}$, where $\mathrm{OR}$ was the estimated odds ratio (8). The confidence limits for the attributable fraction were calculated from the upper and lower limits of the odds ratio.

In one case-referent study, the authors presented differences in mean exposures between the cases and referents. These differences were converted to odds ratios by the method of Greenland (10).

\section{Results}

In our survey of the literature, there were 21 articles and reports that met the established criteria: 15 crosssectional studies in which 32 occupational or exposure groups had been studied (table 1) $(6,11-24)$ and six case-referent studies (table 2) (25-30). All of these studies involved prevalent cases of CTS.

The prevalence of CTS in the different occupational groups varied between 0.6 and $61 \%$ (table 1). The lowest prevalences were noted for industrial workers with low-force, low-repetitive jobs $(0.6 \%)$ and slaughterhouse workers $(1 \%)$ (table 1). The highest prevalences in the cross-sectional studies were noted for grinders, butchers, grocery workers and frozen food factory workers with high-force, high-repetitive gripping jobs, and platers. The estimated effects on CTS in the cross-sectional studies were high for industrial workers with high force and high repetition in their jobs with an odds ratio of 16 and for platers with an odds ratio of 11.0 (table 2 ). In the case-referent studies (table 3) the highest odds ratios were observed for exposures defined as high-level or long-lasting vibration exposure, three different studies showed odds ratios greater than 4 (table 4). An exposure-response relationship for vibration exposure was indicated in both cross-sectional and case-referent studies $(11,25)$. Other occupational factors related to CTS were wrist flexion and extension postures and keying work (table 4).

The impact of occupation or occupationally related exposure on CTS was substantial (table 5). High-force, high-repetition vibration exposure or manual work or both indicated attributable fractions of $80 \%$ or higher in the exposed populations (table 5). Keying had an attributable fraction of $74 \%$ among women working at least $20 \mathrm{~h}$ a week with a keyboard (table 5).

\section{Discussion}

There are numerous sources of estimation error (bias) in epidemiologic studies. In the following discussion

Table 1. Characteristics of the occupational or exposure groups in the included $15 \mathrm{cross}$-sectional studies. (CTS $=$ carpal tunnel syndrome; NA = data not available)

\begin{tabular}{|c|c|c|c|c|c|c|c|}
\hline $\begin{array}{l}\text { Job title, occupational tasks and } \\
\text { study base characteristics }\end{array}$ & Gender & $\mathrm{N}$ & $\begin{array}{l}\text { Mean } \\
\text { age } \\
\text { (years) }\end{array}$ & $\begin{array}{l}\text { Mean } \\
\text { employ- } \\
\text { ment } \\
\text { time } \\
\text { (years) }\end{array}$ & $\begin{array}{l}\text { CTS } \\
\text { preva- } \\
\text { lence } \\
(\%)\end{array}$ & CTS criteria & Study \\
\hline $\begin{array}{l}\text { 1. Platers: stainless steel welding, grinding, } \\
\text { chip-hammering, from employer's list of } 112 \\
\text { platers working as a plater and younger than } \\
55 \text { years of age }\end{array}$ & Male & 89 & 36.7 & 10.1 & 14 & $\begin{array}{l}\text { Paresthesia in hand with median } \\
\text { nerve distribution and a positive } \\
\text { Phalen's or Tinel test }\end{array}$ & $\begin{array}{l}\text { Nilsson et al, } \\
1990 \text { (11) }\end{array}$ \\
\hline $\begin{array}{l}\text { 2. As } 1 \text {, but platers currently exposed to vibra- } \\
\text { tion }\end{array}$ & Male & 71 & 32.4 & 12.2 & 13 & Same as 1 & $\begin{array}{l}\text { Nilsson et al, } \\
1990 \text { (11) }\end{array}$ \\
\hline $\begin{array}{l}\text { 3. Office workers: engineering construction, } \\
\text { supervision and selling; randomiy selected } \\
\text { from employer's list of } 500 \text { office workers } \\
\text { younger than } 55 \text { years of age }\end{array}$ & Male & 61 & 39.4 & - & 2.2 & Same as 1 & $\begin{array}{l}\text { Nilsson et al, } \\
1990(11)\end{array}$ \\
\hline $\begin{array}{l}\text { 4. As } 3 \text {, but office workers with no previous } \\
\text { vibration exposure }\end{array}$ & Male & 45 & 36.7 & - & 1.7 & Same as 1 & $\begin{array}{l}\text { Nilsson et al, } \\
1990 \text { (11) }\end{array}$ \\
\hline $\begin{array}{l}\text { 5. Workers at seven different industrial sites; } \\
39 \text { different jobs classified according to repe- } \\
\text { tition and force requirements for the hands, } \\
\text { high force and high repetition }\end{array}$ & $\begin{array}{l}\text { Male/ } \\
\text { female }\end{array}$ & 157 & 38.0 & 8.3 & 5.6 & $\begin{array}{l}\text { Median nerve paresthesia, noctur- } \\
\text { nal exacerbation, symptoms more } \\
\text { than } 20 \text { times or lasting more than } \\
\text { a week during previous year, onset } \\
\text { of symptoms since beginning of } \\
\text { current job, and job-positive Pha- } \\
\text { len's or Tinel test with exclusion } \\
\text { of thoracic outlet, cervical root, } \\
\text { pronator teres syndrome, rheuma- } \\
\text { toid arthritis and trauma }\end{array}$ & $\begin{array}{l}\text { Silverstein } \\
\text { et al, } 1987 \text { (12) }\end{array}$ \\
\hline $\begin{array}{l}\text { 6. Same as } 5 \text {, but job demands of hands low } \\
\text { force and high repetition }\end{array}$ & $\begin{array}{l}\text { Male/ } \\
\text { female }\end{array}$ & 143 & 40.7 & 8.1 & 2.1 & Same as 5 & $\begin{array}{l}\text { Silverstein } \\
\text { et al, } 1987 \text { (12) }\end{array}$ \\
\hline $\begin{array}{l}\text { 7. Same as } 6 \text {, but job demands of the hands } \\
\text { high force and low repetition }\end{array}$ & $\begin{array}{l}\text { Male/ } \\
\text { female }\end{array}$ & 195 & 38.9 & 7.5 & 1.0 & Same as 5 & $\begin{array}{l}\text { Silverstein } \\
\text { et al, } 1987 \text { (12) }\end{array}$ \\
\hline $\begin{array}{l}\text { 8. Same as } 7 \text {, but job demands of the hands } \\
\text { low force and low repetition }\end{array}$ & $\begin{array}{l}\text { Malel } \\
\text { female }\end{array}$ & 157 & 40.2 & 7.0 & 0.6 & Same as 5 & $\begin{array}{l}\text { Silverstein } \\
\text { et al, } 1987 \text { (12) } \\
\text { (continued) }\end{array}$ \\
\hline
\end{tabular}


Table 1. Continued.

\begin{tabular}{|c|c|c|c|c|c|c|c|}
\hline $\begin{array}{l}\text { Job title, occupational tasks and } \\
\text { study base characteristics }\end{array}$ & Gender & $\mathrm{N}$ & $\begin{array}{l}\text { Mean } \\
\text { age } \\
\text { (years) }\end{array}$ & $\begin{array}{l}\text { Mean } \\
\text { employ- } \\
\text { ment } \\
\text { time } \\
\text { (years) }\end{array}$ & $\begin{array}{l}\text { CTS } \\
\text { preva- } \\
\text { lence } \\
(\%)\end{array}$ & CTS criteria & Study \\
\hline $\begin{array}{l}\text { 9. Forestry workers: chain-saw operators ran- } \\
\text { domly selected from } 186 \text { that had }>500 \mathrm{~h} \text { of } \\
\text { exposure, the total number of employed oper- } \\
\text { ators was } 217\end{array}$ & Male & 79 & 45.3 & - & 25 & $\begin{array}{l}\text { Clinical picture of CTS with distal } \\
\text { latency and motor conduction ve- } \\
\text { locity pathology }\end{array}$ & $\begin{array}{l}\text { Fărkkilä et al, } \\
1988 \text { (13) }\end{array}$ \\
\hline $\begin{array}{l}\text { 10. Butchers working with beef and pork from } \\
\text { two slaughter houses }(N=19) ; 17 \text { participated; } \\
\text { meat cutters not included }\end{array}$ & Male & 17 & 38.8 & 15.3 & 53 & $\begin{array}{l}\text { Median nerve distributed numb- } \\
\text { ness and nerve conduction signs } \\
\text { of median block }\end{array}$ & $\begin{array}{l}\text { Falck \& Aarnio, } \\
1983 \text { (14) }\end{array}$ \\
\hline 11. Same as 10 & Male & 17 & 38.8 & 15.3 & 12 & $\begin{array}{l}\text { Median nerve distributed numb- } \\
\text { ness and Phalen's test positive }\end{array}$ & $\begin{array}{l}\text { Falck \& Aarnio, } \\
1983(14)\end{array}$ \\
\hline $\begin{array}{l}\text { 12. Electricians: work with light- or medium- } \\
\text { weight tools (pliers, screw-drivers, hammers } \\
\text { power tools and cable cutters); all members of } \\
\text { one shop }\end{array}$ & Male & 14 & 49 & 15 & 43 & $\begin{array}{l}\text { Symptoms of paresthesia and } \\
\text { nerve conduction block }\end{array}$ & $\begin{array}{l}\text { Bleecher et al, } \\
1985(15)\end{array}$ \\
\hline $\begin{array}{l}\text { 13. Employees at a meat cutting plant: bi- } \\
\text { manual work involving repetitive grasping and } \\
\text { pulling, in cutting areas room temperature } 50^{\circ} \mathrm{F} \\
\left(11^{\circ} \mathrm{C}\right) \text { with a meat temperature of } 38^{\circ} \mathrm{F}\left(3^{\circ} \mathrm{C}\right)\end{array}$ & Male & 733 & 35.5 & 9.1 & 15 & CTS surgery & $\begin{array}{l}\text { Masear et al, } \\
1986(16)\end{array}$ \\
\hline 14. Same as 13 & Female & 55 & 34.3 & 9.1 & 20 & Same as 13 & $\begin{array}{l}\text { Masear et al, } \\
1986(16)\end{array}$ \\
\hline $\begin{array}{l}\text { 15. All subjects at three workstations at a } \\
\text { poultry-processing company; retrieving a bird } \\
\text { in one position and placing it in a designated } \\
\text { area for further preparation; temperature } 55 \text { to } \\
60^{\circ} \mathrm{F}\left(13 \text { to } 16^{\circ} \mathrm{C}\right) \text {; work categorized as low } \\
\text { force high repetition }\end{array}$ & Female & 35 & NA & NA & 20 & $\begin{array}{l}\text { At least three of following six } \\
\text { symptoms and signs: positive } \\
\text { Tinel sign, positive Phalen sign, } \\
\text { subjective report of morning swell- } \\
\text { ing of hands, three-jaw chuck } \\
\text { pinch reduced from published } \\
\text { norms, a squared wrist dimension, } \\
\text { elevated vibrometer readings com- } \\
\text { pared with the contralateral side in } \\
\text { the median nerve distribution }\end{array}$ & $\begin{array}{l}\text { Muffly-Elsey \& } \\
\text { Flinn-Wagner, } \\
1987(17)\end{array}$ \\
\hline $\begin{array}{l}\text { 16. Slaughterhouse workers in a slaughter- } \\
\text { house with a total of } 119 \text { butchers, meat cut- } \\
\text { ters and meat by-product workers ( } 82 \text { men, } \\
31 \text { women): cutters, butchers meat by-product } \\
\text { workers; meat temperature } 0-7^{\circ} \mathrm{C} \text {; workplace } \\
\text { temperature } 10^{\circ} \mathrm{C}\end{array}$ & $\begin{array}{l}\text { Malel } \\
\text { female }\end{array}$ & 113 & 31.8 & 5.5 & 1 & $\begin{array}{l}\text { Nocturnal paresthesia, positive } \\
\text { Tinel's test, and positive Phalen } \\
\text { test }\end{array}$ & $\begin{array}{l}\text { Viikari-Juntura, } \\
1983(18)\end{array}$ \\
\hline $\begin{array}{l}\text { 17. Garment workers selected from } 214 \text { peo- } \\
\text { ple in a garment shop: women's jackets were } \\
\text { produced, stitcher (sewing machine operator), } \\
\text { finisher (sewing and trimming by hand), under- } \\
\text { presser (ironing by hand), floor work (carrying } \\
\text { bundles), shipping, operation of a facing ma- } \\
\text { chine }\end{array}$ & Female & 162 & 43 & 11 & 6.8 & $\begin{array}{l}\text { Symptoms and positive Tinel test } \\
\text { or Phalen's test }\end{array}$ & $\begin{array}{l}\text { Punnett et al, } \\
1985(19)\end{array}$ \\
\hline 18. Hospital workers in chronic care & Female & 73 & 41 & 5 & 5.5 & $\begin{array}{l}\text { Symptoms and positive Tinel test } \\
\text { or Phalen's test }\end{array}$ & $\begin{array}{l}\text { Punnett, } 1987 \\
\text { (20) }\end{array}$ \\
\hline $\begin{array}{l}\text { 19. Ski-manufacturing workers in a ski-manu- } \\
\text { facturing shop, repetitive jobs }\end{array}$ & $\begin{array}{l}\text { Malel } \\
\text { female }\end{array}$ & 106 & 35 & 5 & 34 & Electrodiagnostics & $\begin{array}{l}\text { Barnhart et al, } \\
1991(21)\end{array}$ \\
\hline $\begin{array}{l}\text { 20. Ski-manufacturing workers in a ski-manu- } \\
\text { facturing shop, nonrepetitive jobs }\end{array}$ & $\begin{array}{l}\text { Malel } \\
\text { female }\end{array}$ & 67 & 41 & 10 & 19 & Same as 19 & $\begin{array}{l}\text { Barnhar et al, } \\
1991 \text { (21) }\end{array}$ \\
\hline $\begin{array}{l}\text { 21. Grocery store workers in a large supermar- } \\
\text { ket with a cluster-of reported CTS; high ex- } \\
\text { posure category }\end{array}$ & $\begin{array}{l}\text { Male/ } \\
\text { female }\end{array}$ & 16 & 35 & 5.2 & 44 & $\begin{array}{l}\text { Symptoms and Tinel test or Pha. } \\
\text { len's test or median sensory defi- } \\
\text { cit of the hand or thenar atrophy }\end{array}$ & $\begin{array}{l}\text { Osorio et al, } \\
1989 \text { (22) }\end{array}$ \\
\hline 22. Same as 21 ; medium exposure category & $\begin{array}{l}\text { Malel } \\
\text { female }\end{array}$ & 30 & 28 & 3.1 & 10 & Same as 21 & $\begin{array}{l}\text { Osorio et al, } \\
1989 \text { (22) }\end{array}$ \\
\hline 23. Postal workers: letter sorting & $\begin{array}{l}\text { Malei } \\
\text { female }\end{array}$ & 79 & 38.4 & 6.6 & 22 & $\begin{array}{l}\text { Symptoms and median sensory } \\
\text { deficit of the hand or a positive } \\
\text { Tinel or Phalen's sign }\end{array}$ & $\begin{array}{l}\text { California } \\
\text { Occupational } \\
\text { Health Pro- } \\
\text { gram, } 1990(23)\end{array}$ \\
\hline $\begin{array}{l}\text { 24. Postal workers: optical character reader } \\
\text { operators }\end{array}$ & $\begin{array}{l}\text { Malel } \\
\text { female }\end{array}$ & 34 & 34.6 & 2.2 & 15 & Same as 23 & $\begin{array}{l}\text { California } \\
\text { Occupational } \\
\text { Health Pro- } \\
\text { gram, } 1990(23)\end{array}$ \\
\hline $\begin{array}{l}\text { 25. Frozen food factory employees: low ex- } \\
\text { posure to cold and low repetitive movements }\end{array}$ & $\begin{array}{l}\text { Male/ } \\
\text { female }\end{array}$ & 49 & 39.9 & 7.3 & 4 & $\begin{array}{l}\text { Symptoms and positive Tinel sign } \\
\text { or Phaien's test }\end{array}$ & $\begin{array}{l}\text { Chiang et al, } \\
1990(24)\end{array}$ \\
\hline $\begin{array}{l}\text { 26. Frozen food factory employees: no ex- } \\
\text { posure to cold but a high degree of repetitive- } \\
\text { ness }\end{array}$ & $\begin{array}{l}\text { Malel } \\
\text { female }\end{array}$ & 37 & 38.0 & 5.5 & 46 & Same as 25 & $\begin{array}{l}\text { Chiang et al, } \\
1990(24)\end{array}$ \\
\hline $\begin{array}{l}\text { 27. Frozen food factory employees: exposed } \\
\text { to cold and a high degree of repetitiveness }\end{array}$ & $\begin{array}{l}\text { Malel } \\
\text { female }\end{array}$ & 121 & 41.5 & 5.2 & 47 & Same as 25 & $\begin{array}{l}\text { Chiang et al, } \\
1990(24)\end{array}$ \\
\hline 28. Grinders (group V) & $\begin{array}{l}\text { Malel } \\
\text { female }\end{array}$ & 23 & NA & NA & 61 & $\begin{array}{l}\text { Maximum latency difference of } 0.4 \\
\text { ms or greater for eight sensory } \\
\text { latencies assessed in consecutive } \\
\text { 1-cm segments of the median } \\
\text { nerve }\end{array}$ & $\begin{array}{l}\text { Nathan et al, } \\
1988(6)\end{array}$ \\
\hline 29. Assembly workers (group III) & $\begin{array}{l}\text { Malel } \\
\text { female }\end{array}$ & 164 & NA & NA & 47 & Same as 28 & $\begin{array}{l}\text { Nathan et al, } \\
1988(6)\end{array}$ \\
\hline 30. Keyboard operators (group II) & $\begin{array}{l}\text { Male/ } \\
\text { female }\end{array}$ & 22 & NA & NA & 27 & Same as 28 & $\begin{array}{l}\text { Nathan et al, } \\
1988(6)\end{array}$ \\
\hline $\begin{array}{l}\text { 31. Administrative and clerical workers } \\
\text { (group I) }\end{array}$ & $\begin{array}{l}\text { Malel } \\
\text { female }\end{array}$ & 147 & NA & NA & 28 & Same as 28 & $\begin{array}{l}\text { Nathan et al, } \\
\text { t988 (6) }\end{array}$ \\
\hline 32. General plant (group IV) & $\begin{array}{l}\text { Malel } \\
\text { female }\end{array}$ & 115 & NA & NA & 38 & Same as 28 & $\begin{array}{l}\text { Nathan et al, } \\
1988(6)\end{array}$ \\
\hline
\end{tabular}


Table 2. Case-referent studies included in the review. (NA = data not available, CTS $=$ carpal tunnel syndrome)

\begin{tabular}{|c|c|c|c|c|c|c|c|}
\hline Case and referent definition & Gender & $\mathbf{N}$ & $\begin{array}{l}\text { Mean } \\
\text { age }\end{array}$ & $\begin{array}{l}\text { Mean } \\
\text { employ- } \\
\text { ment } \\
\text { time }\end{array}$ & $\begin{array}{l}\text { Exposure assessment/ } \\
\text { exposure studied }\end{array}$ & Controlling & Study \\
\hline $\begin{array}{l}\text { 1. Men aged } 20-66 \text { years who were } \\
\text { operated on at one hospital during } \\
1975-1980 \text {; clinical diagnosis and } \\
\text { electrodiagnostic test }\end{array}$ & Male & 34 & NA & NA & $\begin{array}{l}\text { Telephone interview; use of } \\
\text { handheld tools }<1 \text { year, } 1- \\
20 \text { years, or }>20 \text { years }\end{array}$ & Gender, age & $\begin{array}{l}\text { Wieslander } \\
\text { et al, } 1989(25)\end{array}$ \\
\hline $\begin{array}{l}\text { Men from surgical register with one } \\
\text { gallbladder operation for each case } \\
\text { and one varicose veins operation; } \\
\text { general population referents, two for } \\
\text { each case }\end{array}$ & Male & 143 & NA & NA & $\begin{array}{l}\text { Repetitive wrist movements } \\
<1 \text { year, } 1-20 \text { years, or }>20 \\
\text { years; work causing great } \\
\text { load on wrist }<1 \text { year, } 1- \\
20 \text { year, or }>20 \text { year }\end{array}$ & & \\
\hline $\begin{array}{l}\text { II. Persons who had received work- } \\
\text { ers' compensation benefits for wrist- } \\
\text { arm-hand } 1977-1979,16 \text { persons } \\
\text { CTS; medical department diagnosis } \\
1977-1979,14 \text { people identified; } \\
\text { total } 3 \text { men and } 27 \text { women }\end{array}$ & $\begin{array}{l}\text { Malel } \\
\text { female }\end{array}$ & 30 & 43.2 & 5.5 & $\begin{array}{l}\text { Questionnaire; use of vibrat- } \\
\text { ing hand tools; performance } \\
\text { of repetitive motion task; } \\
\text { years on the job }\end{array}$ & $\begin{array}{l}\text { Gender, use of vi- } \\
\text { brating hand tools, } \\
\text { history of gyne- } \\
\text { cologic surgery, } \\
\text { years on the job, } \\
\text { performance of re- } \\
\text { petitive motion }\end{array}$ & $\begin{array}{l}\text { Cannon et al, } \\
1981(26)\end{array}$ \\
\hline $\begin{array}{l}\text { Three referents per subject were ran- } \\
\text { domly selected from persons em. } \\
\text { ployed at the same plant }\end{array}$ & $\begin{array}{l}\text { Malel } \\
\text { female }\end{array}$ & 90 & 44.8 & 11.7 & & & \\
\hline $\begin{array}{l}\text { III. Female sewing machine opera- } \\
\text { tors; medical histories review; "dis- } \\
\text { ease" cases excluded; sewing seat } \\
\text { covers }\end{array}$ & Female & 18 & 33.2 & NA & $\begin{array}{l}\text { Hand force, hand position, } \\
\text { wrist position, pinch force }\end{array}$ & Gender, plant & $\begin{array}{l}\text { Armstrong \& } \\
\text { Chaffin } 1979 \\
\text { (27) }\end{array}$ \\
\hline $\begin{array}{l}\text { Female sewing machine operators, } \\
\text { no CTS }\end{array}$ & Female & 18 & 34.0 & NA & & & \\
\hline $\begin{array}{l}\text { IV. CTS surgery register, Örebro } \\
\text { county }\end{array}$ & Female & 112 & NA & NA & $\begin{array}{l}\text { Repetitive, manual, knitting, } \\
\text { piece work }\end{array}$ & Age, gender & $\begin{array}{l}\text { delaval et al, } \\
1985 \text { (28) }\end{array}$ \\
\hline General population & Female & 199 & NA & NA & Typist keyboard & & \\
\hline V. CTS surgery register & Male & 18 & NA & NA & Vibration exposure & $\begin{array}{l}\text { Age, gender, manu- } \\
\text { al work }\end{array}$ & $\begin{array}{l}\text { Voog et al, } \\
1985(29)\end{array}$ \\
\hline General population & Male & 238 & NA & NA & & & \\
\hline $\begin{array}{l}\text { VI. } 128 \text { consecutive carpal tunnel } \\
\text { syndrome patients from a hospital } \\
\text { and an additional } 28 \text { from a popula- } \\
\text { tion survey }\end{array}$ & $\begin{array}{l}\text { Male/ } \\
\text { female }\end{array}$ & $\begin{array}{r}25 / \\
131\end{array}$ & NA & NA & $\begin{array}{l}\text { Activities with flexed wrist } \\
0-5 \text { years ago; activities } \\
\text { with extended wrist (hours); } \\
\text { activities with extended and } \\
\text { flexed wrist in combination; } \\
\text { pinch, grasp, typing }\end{array}$ & Gender, age & $\begin{array}{l}\text { De Krom et al, } \\
1990(30)\end{array}$ \\
\hline Random population sample & $\begin{array}{l}\text { Male } t \\
\text { female }\end{array}$ & $\begin{array}{l}163 ! \\
310\end{array}$ & NA & NA & & & \\
\hline
\end{tabular}

Table 3. Estimated prevalence of carpal tunnel syndrome and odds ratios for cross-sectional studies with referents. $(95 \%$ $\mathrm{Cl}=95 \%$ confidence interval, NA = data not available)

\begin{tabular}{|c|c|c|c|c|c|c|c|}
\hline \multirow[b]{2}{*}{ Job title or exposure } & \multirow[b]{2}{*}{ Referents } & \multicolumn{3}{|c|}{ Prevalence $(\%)$} & \multirow[b]{2}{*}{$95 \% \mathrm{Cl}$} & \multirow[b]{2}{*}{ Adjustment } & \multirow[b]{2}{*}{ Study } \\
\hline & & $\begin{array}{l}\text { Ex- } \\
\text { posed }\end{array}$ & $\begin{array}{l}\text { Unex. } \\
\text { posed }\end{array}$ & $\begin{array}{l}\text { Odds } \\
\text { ratio }\end{array}$ & & & \\
\hline $\begin{array}{l}\text { High force and high repeti- } \\
\text { tiveness }\end{array}$ & $\begin{array}{l}\text { Low force and low repeti- } \\
\text { tiveness }\end{array}$ & 5.6 & 0.6 & 16 & $1.7-142$ & $\begin{array}{l}\text { Age, gender, plant } \\
\text { years on the job }\end{array}$ & $\begin{array}{l}\text { Silverstein et al, } \\
1987(12)\end{array}$ \\
\hline Platers & Office workers & 14 & 1.7 & 11 & $2.0-62$ & Age & $\begin{array}{l}\text { Nilsson et al, } \\
1990 \text { (11) }\end{array}$ \\
\hline $\begin{array}{l}\text { Frozen food factory workers } \\
\text { exposed to cold and repeti- } \\
\text { tion }\end{array}$ & Frozen food factory workers & 47 & 4.3 & 9.4 & $2.4-37$ & $\begin{array}{l}\text { Gender, age em- } \\
\text { ployment time }\end{array}$ & $\begin{array}{l}\text { Chiang et al, } \\
1990(24)\end{array}$ \\
\hline $\begin{array}{l}\text { Platers with active vibration } \\
\text { exposure }\end{array}$ & Office & 13 & 2.2 & 7.7 & $1.2-50$ & Age & $\begin{array}{l}\text { Nilsson et al, } \\
1990(11)\end{array}$ \\
\hline $\begin{array}{l}\text { Grocery workers: high ex- } \\
\text { posure category }\end{array}$ & $\begin{array}{l}\text { Grocery workers: low or } \\
\text { medium exposure category }\end{array}$ & 44 & 10 & 7.0 & $1.5-33$ & None & $\begin{array}{l}\text { Osorio et al, } \\
1989 \text { (22) }\end{array}$ \\
\hline Vibration & Nonvibration & NA & NA & 5.3 & NA & None & $\begin{array}{l}\text { Silverstein et al, } \\
1987(12)\end{array}$ \\
\hline Grinders & $\begin{array}{l}\text { Administrative and clerical } \\
\text { workers }\end{array}$ & 61 & 28 & 4.0 & $1.6-9.9$ & None & $\begin{array}{l}\text { Nathan et al, } \\
1988(6)\end{array}$ \\
\hline $\begin{array}{l}\text { Low force and high repeti- } \\
\text { tiveness }\end{array}$ & Low force and low repetitive & 2.1 & 0.6 & 2.7 & $0.3-28$ & $\begin{array}{l}\text { Age, gender, plant, } \\
\text { years on the job }\end{array}$ & $\begin{array}{l}\text { Silverstein et al, } \\
1987(12)\end{array}$ \\
\hline Assembly workers & $\begin{array}{l}\text { Administrative and clerical } \\
\text { workers }\end{array}$ & 47 & 28 & 2.3 & $1.4-3.7$ & None & $\begin{array}{l}\text { Nathan et al, } \\
1988(6)\end{array}$ \\
\hline $\begin{array}{l}\text { Ski-manufacturing workers } \\
\text { with repetitive jobs }\end{array}$ & $\begin{array}{l}\text { Ski-manufacturing workers } \\
\text { with nonrepetitive jobs }\end{array}$ & 34 & 19 & 2.3 & $1.1-4.8$ & None & $\begin{array}{l}\text { Barnhart et al, } \\
1991(21)\end{array}$ \\
\hline $\begin{array}{l}\text { Frozen food factory workers } \\
\text { exposed to repetition, no }\end{array}$ & Frozen food factory workers & 41 & 4.3 & 2.2 & $0.2-21$ & $\begin{array}{l}\text { Gender, age, em- } \\
\text { ployment time }\end{array}$ & $\begin{array}{l}\text { Chiang et al, } \\
1990(24)\end{array}$ \\
\hline
\end{tabular}


Table 3. Continued.

\begin{tabular}{|c|c|c|c|c|c|c|c|}
\hline \multirow[b]{2}{*}{ Job title or exposure } & \multirow[b]{2}{*}{ Referents } & \multicolumn{3}{|c|}{ Prevalence $(\%)$} & \multirow[b]{2}{*}{$95 \% \mathrm{Cl}$} & \multirow[b]{2}{*}{ Adjustment } & \multirow[b]{2}{*}{ Study } \\
\hline & & $\begin{array}{c}\text { Ex- } \\
\text { posed }\end{array}$ & $\begin{array}{l}\text { Unex- } \\
\text { posed }\end{array}$ & $\begin{array}{l}\text { Odds } \\
\text { ratio }\end{array}$ & & & \\
\hline Vibration & Nonvibratory exposure & NA & NA & 1.9 & NA & $\begin{array}{l}\text { Force and repeti- } \\
\text { tion }\end{array}$ & $\begin{array}{l}\text { Silverstein et al, } \\
1987 \text { (12) }\end{array}$ \\
\hline $\begin{array}{l}\text { High force and low repeti- } \\
\text { tiveness }\end{array}$ & $\begin{array}{l}\text { Low force and low repeti. } \\
\text { viveness }\end{array}$ & 1.9 & 0.6 & 1.8 & $0.2-21$ & $\begin{array}{l}\text { Age, gender, plant } \\
\text { years on the job }\end{array}$ & $\begin{array}{l}\text { Silverstein et al, } \\
1987(12)\end{array}$ \\
\hline Garment workers & Hospital employees & 6.8 & 5.5 & 1.3 & $0.4-4.1$ & None & $\begin{array}{l}\text { Punnett et al, } \\
1985 \text { (19) }\end{array}$ \\
\hline Keyboard operators & $\begin{array}{l}\text { Administrative and clerical } \\
\text { workers }\end{array}$ & 27 & 28 & 1.0 & $0.3-2.6$ & None & $\begin{array}{l}\text { Nathan et al, } \\
1988(6)\end{array}$ \\
\hline
\end{tabular}

Table 4. Estimated odds ratios for carpal tunnel syndrome in the case-referent studies. ( $95 \% \mathrm{Cl}=95$ percent confidence interval)

\begin{tabular}{|c|c|c|c|c|}
\hline Exposures & $\begin{array}{l}\text { Odds } \\
\text { ratio }\end{array}$ & $95 \% \mathrm{Cl}$ & Adjustment & Study \\
\hline High-level vibration exposure $>10 \mathrm{~h} /$ week & 14 & $5.5-35$ & Gender, age & Voog et al, 1985 (29) \\
\hline Activities with flexed wrist $20-40 \mathrm{~h} /$ week & 8.7 & $3.1-24$ & Gender, age & De Krom et al, $1990(30)$ \\
\hline Use of vibrating hand tools & 7.0 & $3.0-17$ & $\begin{array}{l}\text { Gynecologic surgery, years } \\
\text { on the job, repetitive work } \\
\text { task }\end{array}$ & Cannon et al, 1981 (26) \\
\hline Activities with extended wrist $20-40 \mathrm{~h} /$ week & 5.4 & $1.1-27$ & Gender, age & De Krom et al, 1990 (30) \\
\hline Manual work and vibration & 5.2 & $2.7-9.8$ & Gender & Voog et al, 1985 (29) \\
\hline Vibration exposure $>20$ years & 4.8 & $1.5-16$ & Gender, age & Wieslander et al, 1989 (25) \\
\hline Repetitive movement of wrist $>20$ years & 4.6 & $1.8-12$ & Gender, age & Wieslander et al, 1989 (25) \\
\hline $\begin{array}{l}\text { High-level vibration exposure } 1-10 \mathrm{~h} / \text { week or } \\
\text { medium-level exposure }>10 \mathrm{~h} / \text { week }\end{array}$ & 4.1 & $1.9-8.7$ & Gender, age & Voog et al, $1985(29)$ \\
\hline Typist keyboard & 3.8 & $1.3-11$ & Gender, age & de Laval et al, 1985 (28) \\
\hline Vibration exposure, medium level $1-10 \mathrm{~h} /$ week & 3.2 & $1.2-8.2$ & Gender, age & Voog et al, 1985 (29) \\
\hline Activities with flexed wrist $8-19 \mathrm{~h} /$ week & 3.0 & $1.8-4.9$ & Gender, age & De Krom et al, $1990(30)$ \\
\hline Activities with extended wrist $8-19 \mathrm{~h} /$ week & 2.3 & $1.0-5.2$ & Gender, age & De Krom et al, $1990(30)$ \\
\hline Repetitive work task & 2.2 & $1.3-3.6$ & Gender, age & de Laval et at, 1985 (28) \\
\hline Repetitive motion tasks & 2.1 & $0.86-5.3$ & $\begin{array}{l}\text { Gynecologic surgery, vibrat- } \\
\text { ing tools, years on the job }\end{array}$ & Cannon et al, $1981(26)$ \\
\hline Pinch force & $2.0^{a}$ & $1.6-2.5^{\mathrm{a}}$ & None & $\begin{array}{l}\text { Armstrong \& Chaffin, } \\
1979(27)\end{array}$ \\
\hline Manual work & 1.9 & $1.0-3.5$ & Gender, age & Voog et al, 1985 (29) \\
\hline Repetitive movement of wrist $1-20$ years & 1.5 & $0.5-4.4$ & Gender, age & Wieslander et al, 1989 (25) \\
\hline Activities with flexed wrist $1-7 \mathrm{~h} /$ week & 1.5 & $1.3-1.9$ & Gender, age & De Krom et al, $1990(30)$ \\
\hline Activities with extended wrist $1-7 \mathrm{~h} /$ week & 1.4 & $1.0-1.9$ & Gender, age & De Krom et al, $1990(30)$ \\
\hline Repetitive manual tasks, $50-65$ years of age & 1.4 & $0.6-3.2$ & Gender, age & de Laval et al, 1985 (28) \\
\hline Activities with extended and flexed wrist $20-40 \mathrm{~h} /$ week & 1.4 & $0.7-2.9$ & Gender, age & De Krom et al, $1990(30)$ \\
\hline Activities with extended and flexed wrist $8-19 \mathrm{~h} /$ week & 1.2 & $0.8-1.7$ & Gender, age & De Krom et al, $1990(30)$ \\
\hline Activities with extended and flexed wrist $1-7 \mathrm{~h} /$ week & 1.1 & $0.9-1.2$ & Gender, age & De Krom et al, $1990(30)$ \\
\hline Hand force & $1.05^{a}$ & $0.95-1.15^{a}$ & None & $\begin{array}{l}\text { Armstrong \& Chaffin, } \\
1979 \text { (27) }\end{array}$ \\
\hline Pinch grasp $1-7 \mathrm{~h} /$ week & 0.9 & $0.8-1.1$ & Gender, age & De Krom et al, $1990(30)$ \\
\hline Typing $1-7$ h/week & 0.9 & $0.6-1.4$ & Gender, age & De Krom et al, $1990(30)$ \\
\hline Years on the job & 0.89 & $0.81-0.97$ & $\begin{array}{l}\text { Gynecologic surgery, vibrat- } \\
\text { ing tools, repetitive work } \\
\text { task }\end{array}$ & Cannon et al, 1981 (26) \\
\hline Pinch grasp $8-19$ h/week & 0.8 & $0.5-1.3$ & Gender, age & De Krom et al, $1990(30)$ \\
\hline Typing $8-19 \mathrm{~h} /$ week & 0.8 & $0.3-2.5$ & Gender, age & De Krom et al, $1990(30)$ \\
\hline Pinch grasp $20-40 \mathrm{~h} /$ week & 0.7 & $0.3-1.6$ & Gender, age & De Krom et al, $1990(30)$ \\
\hline Typing $20-40 \mathrm{~h} /$ week & 0.7 & $0.1-6.0$ & Gender, age & De Krom et al, $1990(30)$ \\
\hline
\end{tabular}

a Computed from mean values and standard deviation according to Greenland (10).

we evaluate the relevant CTS literature in terms of the following four major threats to (internal) validity: (i) selection bias and temporal ambiguity, (ii) problems of case definition and identification, (iii) problems of exposure definition and measurement, (iv) and con- founding. In addition, we consider several other criteria that reflect our ability to make scientific generalizations about causal associations $(8,31-34)$. Although we recognize the limitations in defining any set of causal criteria (31), we discuss the five criteria that 
Table 5. Attributable fractions in the exposed population (AFE) for exposures and job titles above 0.50 in the diagnosis of carpal tunnel syndrome $(95 \% \mathrm{Cl}=95 \%$ confidence interval, $\mathrm{NA}=$ data not available)

\begin{tabular}{|c|c|c|c|}
\hline Exposures/job titles & AFE & $95 \% \mathrm{Cl}$ & Study \\
\hline High force and high repetitiveness & 0.94 & $0.41-0.99$ & Silverstein et al, 1987 (12) \\
\hline High level vibration exposure $>10 \mathrm{~h} /$ week & 0.93 & $0.82-0.97$ & Voog et al, 1985 (29) \\
\hline Platers & 0.91 & $0.50-0.98$ & Nilsson et al, 1990 (11) \\
\hline Frozen food factory workers exposed to cold and repetition & 0.89 & $0.58-0.97$ & Chiang et al, 1990 (24) \\
\hline Activities with flexed wrist $20-40 \mathrm{~h} /$ week & 0.89 & $0.68-0.96$ & De Krom et al, $1990(30)$ \\
\hline Grocery workers high exposure category & 0.86 & $0.33-0.97$ & Osorio et al, 1989 (22) \\
\hline Use of vibrating hand tools & 0.86 & $0.67-0.94$ & Cannon et al, $1981(26)$ \\
\hline Vibration exposure & 0.81 & NA & Silverstein et al, 1987 (12) \\
\hline Manual work and vibration & 0.81 & $0.63-0.90$ & Voog et al, $1985(29)$ \\
\hline Activities with extended wrist $20-40 \mathrm{~h} /$ week & 0.81 & $0.09-0.96$ & De Krom et al, $1990(30)$ \\
\hline Vibration exposure $>20$ years & 0.79 & $0.33-0.94$ & Wieslander et al, 1989 (25) \\
\hline Repetitive movement of wrist & 0.78 & $0.44-0.92$ & Wieslander et al, 1989 (25) \\
\hline $\begin{array}{l}\text { High-level vibration exposure } 1-10 \mathrm{~h} / \text { week or medium-level } \\
\text { exposure }>10 \mathrm{~h} / \text { week }\end{array}$ & 0.77 & $0.47-0.89$ & Voog et al, 1985 (29) \\
\hline Grinders & 0.75 & $0.38-0.90$ & Nathan et al, $1988(6)$ \\
\hline Typist, keyboard & 0.74 & $0.23-0.91$ & Voog et al, 1985 (29) \\
\hline Medium-level vibration exposure $1-10 \mathrm{~h} /$ week & 0.69 & $0.17-0.88$ & Voog et al, $1985(29)$ \\
\hline Activities with flexed wrist $8-19 \mathrm{~h} /$ week & 0.67 & $0.44-0.80$ & De Krom et al, $1990(29)$ \\
\hline Assembly work & 0.57 & $0.29-0.73$ & Nathan et al, $1988(6)$ \\
\hline Low force and high repetitiveness & 0.63 & $0.70-0.96$ & Silverstein et al, 1987 (12) \\
\hline Ski-manufacturing workers with repetitive jobs & 0.57 & $0.09-0.79$ & Barnhart et al, 1991 (21) \\
\hline Activities with extended wrist $8-19 \mathrm{~h} /$ week & 0.57 & $0-0.81$ & De Krom et al, $1990(30)$ \\
\hline Repetitive work task & 0.55 & $0.23-0.72$ & de Laval et al, $1985(28)$ \\
\hline Frozen food factory workers exposed repetition & 0.55 & $0.80-0.95$ & Chiang et al, 1990 (24) \\
\hline Repetitive motion task & 0.52 & $0.17-81$ & Cannon et al, $1981(26)$ \\
\hline Pinch force & 0.50 & $0.38-0.60$ & Armstrong \& Chaffin, 1979 (27) \\
\hline
\end{tabular}

we believe are the most relevant to generalizing about the possible effect of physical work exposure on the occurrence of CTS: strength of association, consistency of results, coherence of evidence, experimental and laboratory support, and literature availability.

\section{Selection bias and temporal ambiguity}

A potential limitation of prevalence studies is that the time of disease onset among study cases is usually not known. Consequently, we cannot be sure that the exposure preceded disease occurrence, especially when the exposure is measured at the same time the disease status is observed. Even retrospective measurement of the exposure may not rectify this problem of "temporal ambiguity," since we seldom know the exact time of disease or symptom onset.

The problem of temporal ambiguity is the most likely to threaten the validity of a prevalence study when the disease or related symptoms can affect exposure status or when the disease can affect subject selection differentially through exposure status. In occupational studies, the latter phenomenon is a secondary form of the well-known "healthy-worker effect"; that is, exposed workers who develop symptoms of CTS or other conditions (in part because they are exposed) leave their jobs and are not selected for future studies of working populations. This type of selection problem, there- fore, will probably lead to a negative bias in the estimation of the effect of physical work exposures on CTS. Thus prevalence findings are likely to underestimate the true exposure effect.

\section{Problems of case definition and identification}

In all of the studies selected for this review, the diagnostic criteria for CTS included both symptoms and signs. In most of the studies it was not clear what quality of symptoms or combination of symptoms was required to meet the case criteria. Median nerve distribution of symptoms was a case criterion used by most of the studies. The criteria for intensity and duration of hand symptoms to meet the case criteria may have differed among the reviewed studies. In studies in which the sign was a positive Phalen's or Tinel test, however, there may have been undetected cases in comparison with studies in which nerve conduction measurements were performed, and the sign was defined as median nerve conduction block. It has been reported that the sensitivity of the Phalen's or Tinel test is only $60-67 \%$ in comparison with electrophysiological nerve conduction block as the "gold standard" (35). De Krom et al (36) claimed that provocative tests do not distinguish between CTS and other causes of nocturnal hand complaints. This possibility could explain some of the variability between similar occupational 
groups. We would urge scientists to use electrodiagnostic tests for CTS for case definition in future studies of CTS.

\section{Problems of exposure definition and measurement}

In the cross-sectional studies, exposure was defined by job title according to the employer's records. In the case-referent studies, the exposure was usually obtained by questionnaire or interview. There was no study in which direct exposure measurements were performed in the workplace for all of the study persons. Job titles and questionnaire data are crude indicators of exposure $(37,38)$. The variability of exposure definition (eg, job title criteria, posture and movement criteria) is probably great across different studies.

\section{Confounding and individual susceptibility}

Individual risk factors (individual indicators of susceptibility) can act as confounders of exposure effects. The most important confounders are age, gender, anthropometry, and other diseases.

The stress capacity of different tissues decreases with age, causing a shift of the stress-strain curve. The normal reparative and wound healing process is also slower with age. The duration of exposure is related to age. There is yet no study that has shown age as a risk factor for CTS when the duration of exposure is controlled.

The incidence rate of CTS is related to gender. A male-to-female ratio of 1:3 was described in a population study (39). There is yet no evidence for an increased female susceptibility for work-related CTS when exposure is controlled for. In a population-based incidence study of occupational CTS, the male-tofemale ratio was 1.2:1 (40). In the study, by Silverstein et al (12), of CTS among industrial workers, no difference was observed between genders when exposure factors were controlled.

Carpal canal size is a controversial risk factor for CTS. There are different studies linking CTS with both small and large areas $(15,41)$. Other disorders associated with CTS are diabetes mellitus, myxedema, acromegaly, amyloidosis, fracture of the forearm, and the like. There is one report of a CTS prevalence of $11 \%$ for diabetes mellitus patients (42). For pregnant women an incidence of CTS of up to $25 \%$ has been reported $(43,44)$.

Most of these studies have taken into account possible confounding by age and gender. Would the other individual risk factors influence the inference of occupation and job tasks as risk factors for CTS? For example, Juntunen et al (45) questioned the association between vibration exposure and CTS, claiming that patients with neuropathic diatheses, who are at greater risk for CTS, tend to be selected into groups of patients with vibration syndrome. This phenomenon could be due to a detection problem (ie, workers with vibration exposure are likely to get more medi- cal attention because of white fingers than unexposed workers do). If so, the frequency of CTS would be higher among exposed workers because of earlier diagnosis or detection in this group. However, this detection bias was not present in the cross-sectional studies in which the same diagnostic procedures were used for both the study and reference groups. Nevertheless, it is not likely that workers with neuropathic diatheses would be exposed to physical work load such as repetitive forceful gripping and vibration to a greater extent than would workers without this susceptibility. It is more likely, we believe, that the most susceptible workers would seek jobs with less physical workload exposure. Although exposure to physical workload factors can result in CTS as an early manifestation of a neurologic disorder in some workers (46), the frequency of such disorders is probably very low in worker populations $(17,47,48)$.

\section{Strength of association}

The strength of association in the reviewed studies was generally high. There were three different studies reporting odds ratios greater than 10 for (i) high repetition and high force in the hands, (ii) vibration exposure, and (iii) occupation as a plater. Such large effects cannot be easily explained by any sources of bias (eg, a confounder), especially biases that were not evident to the investigators. Nevertheless it should be noted that even strong observed associations are not incompatible with spurious results.

\section{Consistency of results}

There was a surprising consistency of observed effects across the different cross-sectional studies, as well as between the case-referent studies and the cross-sectional studies. Occupational tasks or job titles associated with vibration exposure, repetitive hand movements, and forceful grips were reported in both the cross-sectional and case-referent studies as risk factors for CTS. Vibration exposure is probably an indicator of exposure to forceful repetitive gripping. In the study by Silverstein et al (12), the crude odds ratio for exposure to vibration was 5.3, but the odds ratio adjusted for high force and high repetition was only 1.9 (table 3 ). One inconsistency was noted, however. Butchers were reported to have a CTS prevalence of $53 \%$ in the study by Falck \& Aarnio (14), but the 113 slaughterhouse workers (including 38 butchers) studied by Viikari-Juntura (18) had a reported prevalence of only $1 \%$ (one case with the job title cutter). One explanation for this inconsistency is that CTS was defined by symptoms and nerve conduction measurements, which may be a more sensitive indicator of CTS, in the Falck \& Aarnio study. If one considers only symptoms and a positive Phalen's test in the Falck \& Aarnio study, the prevalence would drop from 53 to $12 \%$ (exact $95 \%$ confidence interval 1-36) (14). Furthermore, the secondary healthy worker selection may have been 
stronger in the Helsinki area, it being a metropolitan area with more job opportunities than in the smaller town of Pori where Falck \& Aarnio performed their study. Thus the inconsistency could have been due to different diagnostic criteria, sociodemographic factors, or chance.

The issue of temporality, which means that the exposure precedes the onset of the disorder, was not demonstrated in all of the studies. In the investigation by Nilsson et al (11) the observed latency (duration between onset of exposure and symptoms) for CTS was reported. In the study by Silverstein et al (12), only disorders that developed after the onset of exposure were considered. In most of the other studies it was not clear whether all registered exposure preceded the onset of symptoms. In future studies of CTS we would urge investigators to explore the temporality of CTS and to establish exposure-latency time relationships.

\section{Coherence of evidence}

An exposure-response relationship was reported in several studies. For example, Silverstein et al (12) found that both repetition and force were predictors of CTS and, if both exposure factors were present, the risk of CTS was even greater. Repetition and force can be regarded as two components of biomechanical stress exposure. In the study comparing platers with office workers, the risk of CTS increased with the number of years exposed to vibration (11). In the case-referent study of Wieslander et al (25), there were progressive trends in the odds ratios with the number of years of use of handheld vibrating tools and repetitive movements of the wrist. In the studies of Silverstein et al (12), Cannon et al (26), and Chiang et al (24), however, the number of years on the job was not a predictor of CTS. One explanation for this inconsistency may be that only certain individuals are at risk of developing CTS and the latency for these individuals is relatively short when they are exposed to hazardous physical work load. Thus cumulative exposure may not be an important predictor of CTS.

\section{Experimental and laboratory support}

There are experimental studies on humans that support the causal relationship between ergonomic factors and CTS. Exposures are likely to affect the median nerve both directly through mechanical stress (eg, stretching and compression) and indirectly through ischemia causing paresthesia or a nerve conduction block. A localized pressure greater than $50 \mathrm{~mm} \mathrm{Hg}$ ( $\approx 6666 \mathrm{~Pa}$ ) in the carpal tunnel or a tourniquet with a pressure higher than systolic around the upper arm will cause a conduction block in the median nerve at the wrist (44). Extreme flexing or extension of the wrist causes an increase in pressure in the carpal tunnel that can affect the blood perfusion of the median nerve (49, 50). The histopathology of CTS shows coherence with the epidemiologic evidence for physical work load as a risk factor. Microscopic studies of tissues in the carpal tunnel in wrist specimens reveal changes (eg, an increase in epineurium density) suggesting that repeated exertions with a flexed or extended wrist are involved in the etiology of CTS (51). These changes relate to mechanical pressure and perfusion of the median nerve.

\section{Literature availability}

Most of the studies considered in this review were cross-sectional investigations. All of the case-referent studies dealt with prevalent cases. There were no prospective cohort investigations. Furthermore, a review of the association between occupation and CTS is likely to be distorted by the fact that there is a tendency to publish positive findings rather than negative or equivocal ones. However, since data concerning the epidemiology of work-related musculoskeletal disorders is sparse, reports of negative or equivocal findings of CTS epidemiology are probably welcome in the journals. An example of an equivocal report is the study of slaughterhouse workers by Viikari-Juntura (18), who found only one case of CTS among 117 workers.

\section{Concluding remarks}

On the basis of epidemiologic and other evidence, we conclude that exposure to physical workload factors such as repetitive and forceful gripping is probably a major risk factor for CTS in several types of worker populations. At least $50 \%$, and as high as $90 \%$, of all CTS cases in these exposed populations appear to be attributable to physical work load.

\section{Acknowledgments}

Financial support from the Swedish Work Environment Fund is gratefully acknowledged.

\section{References}

1. Hadler NM. Cumulative trauma disorders an iatrogenic concept. J Occup Med 1990;32:38-41.

2. Hadler NM. Work-related disorders of the upper extremity: part II. occupational problems in medical practice. Wilmington, DE: MPI Medical Publications Inc, 1989;4:2-11.

3. Gerr F, Letz R, Landrigan PJ. Upper-extremity musculoskeletal disorders of occupational origin. Ann Rev Publ Health 1991;12:543-66.

4. Stock SR. Workplace ergonomic factors and the development of musculoskeletal disorders of the neck and upper limbs a meta-analysis. Am J Ind Med 1991;19: 87-107.

5. Gelberman RH, Rydevik BL, Pess GM, Szabo RM, Lundborg G. Carpal tunnel syndrome a scientific basis for clinical care. Orthop Clin North Am 1988;19:11524.

6. Nathan PA, Meadows KD, Doyle LS. Occupation as a risk factor for impaired sensory conduction of the median nerve at the carpal tunnel. J Hand Surg 1988; 13:167-170. 
7. Gardner MJ, Altman DG. Statistics with confidence. London: British Medical Journal, 1989:144.

8. Kleinbaum DG, Kupper LL, Morgenstern H. Epidemiologic research: principles and quantitative methods. Belmont: Lifetime Learning Publications, 1982.

9. Newman SC. Odds ratio estimation in a steady-state population. J Clin Epidemiol 1988;41:59-65.

10. Greenland S. Quantitative methods in the review of epidemiologic literature. Epidemiol Rev 1987;9:1-30.

11. Nilsson $T$, Hagberg $M$, Burström L, Lundström $R$. Prevalence and odds ratios of numbness and carpal tunnel syndrome in different exposure categories of platers. In: Okada A, Dupuis WTH, ed. Hand-arm vibration. Kanazawa, Japan: Kyoei Press Co, 1990:235-9.

12. Silverstein BA, Fine LJ, Armstrong TJ. Occupational factors and carpal tunnel syndrome. Am J Ind Med 1987;11:343-58.

13. Färkkilä M, Pyykkö I, Jäntti V, Aatola S, Starck J, Korhonen O. Forestry workers exposed to vibration a neurological study. Br J Ind Med 1988;45:188-92.

14. Falck B, Aarnio P. Left-sided carpal tunnel syndrome in butchers. Scand J Work Environ Health 1983;9: 291-7.

15. Bleecker ML, Bohlman M, Moreland R, Tipton A. Carpal tunnel syndrome: role of carpal canal size. Neurology 1985;35:1599-1604.

16. Masear VR, Hayes JM, Hyde AG. An industrial cause of carpal tunnel syndrome. J Hand Surg 1986;11A: 222-7.

17. Muffly-Elsey D, Flinn-Wagner S. Proposed screening tool for the detection of cumulative trauma disorders of the upper extremity. J Hand Surg 1987;12A:931-5.

18. Viikari-Juntura E. Neck and upper limb disorders among slaughterhouse workers: an epidemiologic and clinical study. Scand J Work Environ Health 1983;9:283-90.

19. Punett L, Robins JM, Wegman DH, Keyserling WM. Soft tissue disorders in the upper limbs of female garment workers. Scand J Work Environ Health 1985;11: $417-25$.

20. Punett L. Upper extremity musculoskeletal disorders in hospital workers. J Hand Surg 1987;12A:858-62.

21. Barnhart S, Demers PA, Miller M, Longstreth WT Jr, Rosenstock L. Carpal tunnel syndrome among ski manufacturing workers. Scand J Work Environ Health 1991;17:46-52.

22. Osorio AM, Ames R, Jones J. Carpal tunnel syndrome among grocery store workers. Berkeley, CA: California Occupational Health Program, California Department of Health Services, 1989:61. (Field investigation FI-86-005.)

23. California Occupational Health Program. Carpal tunnel syndrome among postal machine operators. Berkeley, CA: California Occupational Health Program, 1991:44. (Field investigation report FT-86-008.)

24. Chiang H-C, Chen S-S, Yu H-S, Ko Y-C. The occurrence of carpal tunnel syndrome in frozen food factory employees. Kaohsiung J Med Sci 1990;6:73-80.

25. Wieslander G, Norbäck D, Göthe C-J, Juhlin L. Carpal tunnel syndrome (CTS) and exposure to vibration, repetitive wrist movements, and heavy manual work a case-referent study. Br J Ind Med 1989;46:43-7.

26. Cannon LJ, Bernacki EJ, Walter SD. Personal and occupational factors associated with carpal tunnel syndrome. J Occup Med 1981;23:255-8.

27. Armstrong TJ, Chaffin DB. Carpal tunnel syndrome and selected personal attributes. J Occup Med 1979;21: $481-6$.

28. de Laval J, Voog L, Ahlborg G, Holm Glad J. Distal kompression av nervus medianus och ergonomiska belastningar hos kvinnor [Compression of the median nerve at the wrist and ergonomic exposure in women]. Stockholm: Swedish Work Environment Fund, 1985. (Reportproject 83-0121.)
29. Voog L, Ahlborg G, de Laval J, Holm Glad J. Distal kompression av nervus medianus och arbete med vibrerande handverktyg [Compression of the median nerve at the wrist and work with vibrating hand-held tools]. Stockholm: Swedish Work Environment Fund, 1985. (Report project 82-0545.)

30. De Krom MC, D KA, Knipschild PG, Spaans F. Risk factors for carpal tunnel syndrome. Am J Epidemiol 1990;132:1102-10.

31. Rothman KJ, ed. Causal inference. Chestnut Hill, MA: Epidemiology Resources Inc, 1988.

32. Hill AB. The environment and disease: association or causation? Proc R Soc Med 1965;58:295-300.

33. Rothman KJ. Modern epidemiology. Boston, MA: Little, Brown and Company, 1987.

34. Susser M. What is cause and how do we know one? A grammar for pragmatic epidemiology. Am J Epidemiol 1991;133:635-48.

35. Heller L, Ring H, Costeff H, Solzi P. Evaluation of Tinel's and Phalen's signs in diagnosis of the carpal tunnel syndrome. Eur Neurol 1986;25:40-2.

36. De Krom MC, Knipschild PG, Kester AD, Spaans F. Efficacy of provocative tests for diagnosis of carpal tunnel syndrome. Lancet 1990;335:393-5.

37. Hagberg M. Occupational musculoskeletal disorders a new epidemiologic challenge. In: Hogstedt C, Reuterwall $\mathrm{C}$, ed. Epidemiology in occupational health. Amsterdam: Elsevier Science Publisher, 1988:15-26.

38. Hagberg M. Exposure variables in ergonomic epidemiology. Am J Ind Med 1992;21:91-100.

39. Stevens JC, Sun S, Beard CM, O' Fallon WM, Kurland LT. Carpal tunnel syndrome in Rochester, Minnesota, 1961 to 1980 . Neurology $1988 ; 38: 134-8$.

40. Franklin GM, Haug J, Heyer N, Checkoway H. Occupational carpal tunnel syndrome in Washington State, 1984-1988. Am J Public Health 1991;81:741-6.

41. Winn FJ, Habes DJ. Carpal tunnel area as risk factor for carpal tunnel syndrome. Muscle Nerve 1990;13:25458.

42. Comi G, Lozza L, Galardi G, Ghilardi MF, Medaglini $\mathrm{S}$, Canal N. Presence of carpal tunnel syndrome in diabetics: effect of age, sex, diabetes duration and polyneuropathy. Actal Diabetol Lat 1985;22:259-62.

43. Voitk AJ, Mueller JC, Farlinger DE, Johnston RU. Carpal tunnel syndrome in pregnancy. Can Med Assoc J $1983 ; 128: 277-81$.

44. Lundborg G. Nerve injury and repair. Edinburgh: Churchill Livingstone, 1988.

45. Juntunen J, Matikainen E, Seppäläinen AM, Laine A. Peripheral neuropathy and vibration syndrome. Int Arch Occup Environ Health 1983;52:17-24.

46. Hagberg M, Almay B, Kolmodin-Hedman B, Zetterlund B. Vibration exposure - a modifier of the onset of amyloid polyneuropathy: a case report. Scand J Work Environ Health 1986;12:277-9.

47. Hagberg M, Nyström $\AA$, Zetterlund B. Recovery from symptoms after carpal tunnel surgery in males in relation to vibration exposure. J Hand Surg 1991;16A: $66-71$.

48. Mühlau G BR Kunath H. Carpal tunnel syndrome course and prognosis. J Neurol 1984;231:83-6.

49. Werner CO, Elmquist D, Ohlin T. Pressure and nerve lesions in the carpal tunnel. Acta Orthop Scand 1983; $54: 312-6$.

50. Gelberman RH, Hergenroeder PT, Hagens AR, Lundborg GN, Akeson WH. The carpal tunnel syndrome a study of carpal canal pressures. J Bone Jt Surg 1981;63A:380-3.

51. Armstrong TJ, Castelli WA, Evans FG, Diaz-Perez R. Some histological changes in the carpal tunnel contents and their biomechanical implications. J Occup Med 1984;26:197-201.

Received for publication: 13 February 1992 